\title{
Extended Antiepileptic Drug Prophylaxis and Late Onset Seizures in Aneurysmal Subarachnoid Hemorrhage
}

\author{
Wen Hao Low* ${ }^{*}$, Qing Yuan Goh, Miqi Mavis Teo \\ Division of Anesthesiology and Perioperative Sciences, Singapore General Hospital, Singapore \\ Email: *low.wen.hao@singhealth.com.sg
}

How to cite this paper: Low, W.H., Goh, Q.Y. and Teo, M.M. (2019) Extended Antiepileptic Drug Prophylaxis and Late Onset Seizures in Aneurysmal Subarachnoid Hemorrhage. Open Journal of Modern Neurosurgery, 9, 401-409.

https://doi.org/10.4236/ojmn.2019.94037

Received: September 2, 2019

Accepted: September 24, 2019

Published: September 27, 2019

Copyright $\odot 2019$ by author(s) and Scientific Research Publishing Inc. This work is licensed under the Creative Commons Attribution International License (CC BY 4.0).

http://creativecommons.org/licenses/by/4.0/

\begin{abstract}
Background: The indication and optimal duration of antiepileptic drug (AED) prophylaxis after aneurysmal subarachnoid hemorrhage (SAH) remains controversial. Our institution practices routine seizure prophylaxis for variable durations at the discretion of the neurosurgeon and neuro-intensivist. Given the propensity of late onset seizures to progress to chronic epilepsy, we sought to investigate the efficacy of extended AED prophylaxis in reducing the risk of late seizures. Methods: This retrospective study analyzed 36 patients who were admitted to our neurosurgical intensive care unit (NICU) over a 2-year period (1st November 2015 to 31st October 2017). All hospital admissions records, electronic medication records as well as outpatient visits up to 1 year were reviewed. Late onset seizures were defined as seizures occurring more than 7 days post-intervention (or presentation if no intervention was performed) up to 1 year of follow-up. Results: Majority of the patients received Levetiracetam (94\%) as seizure prophylaxis and late onset seizures occurred in $6(17 \%)$ of the patients. Those patients who received a greater proportion of in-patient stay with AED prophylaxis had a statistically significant lower risk of developing late seizures ( $O R=0.964,95 \%, p=0.02)$. Although the value tended towards benefit $(\mathrm{OR}=0.382)$ for AED $>21$ days in-hospital, the result was not statistically significant ( $p=0.307)$. Conclusion: An extended duration of AED prophylaxis, in particular Levetiracetam, may confer some benefit in reducing risk of developing late seizures. However, the optimal duration of therapy is yet to be determined and further large multi-centered randomized studies are necessary.
\end{abstract}

\section{Keywords}

Antiepileptic, Seizure Prophylaxis, Aneursymal Subarachnoid Hemorrhage 


\section{Introduction}

Seizures as a complication of subarachnoid hemorrhage (SAH) from aneurysmal rupture have been reported to have an incidence ranging from $10 \%$ to $26 \%$ [1] [2] [3]. It is an important clinical concern with potentially catastrophic consequences from re-rupture of an unsecured lesion [4]. Seizures are also associated with immediate complications of increased intra-cranial pressure and metabolic stress as well as late complications such as chronic epilepsy and poor outcome 30 to 90 days from aneurysm rupture [3] [5].

Onset seizures are usually defined as seizures occurring at the time of and within $12-24$ hours of aneurysm rupture and have been reported at a rate of about $10 \%$ [5]. Although most series have not found onset seizures to be independently associated with poor outcome or with chronic epilepsy [6], there has been an association in one study [5]. Early seizures (within 1 week from intervention) have an incidence of $2.3 \%$ and late seizures ( $>1$ week from intervention) occur at a rate of 5.5\% [7]. Of note, $53.6 \%$ of patients who developed a late seizure progressed to chronic epilepsy [1].

Despite more than three decades of research to minimize seizure load, the indication and optimal duration of antiepileptic drug (AED) prophylaxis remains a subject of much controversy. Due to concerns about safety of routine AED prophylaxis, primarily Phenytoin, related to neurological deterioration, vasospasms and poorer outcome [8] [9], there was a paradigm shift against AED prophylaxis with some institutions recommending no prophylaxis [10].

The advent of Levetiracetam with superior tolerability and favorable pharmacokinetic profile has brought about renewed support in the practice of AED prophylaxis [11]. A recent survey sent to 25 US centers with high volume aneurysmal SAH cases revealed $52 \%$ of respondents endorsing utility of seizure prophylaxis with $94 \%$ using Levetiracetam as the first-line medication [12]. Duration of AED prophylaxis was highly varied, ranging from 1 day to 6 weeks following SAH with most clinicians preferring a duration of 7 to 21 days.

In our center, routine seizure prophylaxis is being practiced with close follow-up of patients for a year after SAH. This presents a unique dataset where extended AED therapy and late onset seizures can be examined. Therefore, we conceptualized this study to review the effect of the duration of AED prophylaxis on the incidence of late onset seizures occurring more than 7 days post-intervention up to 1 year. Given the propensity for late seizures to progress to chronic epilepsy [1], we believe they may be amenable to extended AED prophylaxis. We also examined the factors that may contribute to late onset seizures that may form part of a set of criteria for recommendation of extended AED prophylaxis.

\section{Methods}

We retrospectively analyzed patients who were admitted to our NICU with aneurysmal SAH over a 2-year period (1st November 2015 to 31st October 2017). 
Institutional Review Board approved this study (CIRB 2018/2417) with waiver of informed consent. We identified these patients using the hospital International Classification of Diseases (ICD) discharge diagnosis of SAH [13]. Patients were excluded if SAH was secondary to non-aneurysmal causes such as trauma or spontaneous intraparenchymal hemorrhage. Patients with pre-existing seizure disorder or death within 72 hours were also excluded. All hospital admission records, electronic medication records as well as outpatient visits up to 1 year were retrospectively studied. Our electronic database captures nationwide data and all outpatient as well as emergency visits will be recorded. Baseline demographic information (age, sex, co-morbidities), clinical characteristics at presentation (GCS recorded, modified Fisher grade and location of aneurysm), type of intervention (clipping, coiling or none), evidence of vasospasm, length of hospital stay and GCS at discharge were recorded. Seizures were documented according to their time of onset: [1] [2]

1) Onset seizure-within 12 - 24 hours of presentation;

2) Early seizure-within 7 days of intervention or presentation if no intervention was performed;

3) Late seizure-more than 7 days post-intervention or presentation, up to 1 year of follow-up.

Seizures observed in the pre-hospital setting at initial presentation or during the follow-up period by family members or caregivers, paramedics and ED staff were recorded. Seizures were diagnosed clinically or by EEG as inpatients.

Choice of AED, including loading dose and duration of therapy was left to the discretion of the attending intensivist and neurosurgeon. Levetiracetam was the predominant choice and was initiated at a loading dose of IV $1000 \mathrm{mg}$ followed by a 500 to $750 \mathrm{mg}$ BD maintenance dose. Phenytoin was administered at IV 1 gm loading with $100 \mathrm{mg}$ every 8 hours.

Statistical analysis was performed using IBM SPSS Statistics (Version 26) with. Results of univariate logistic regression are presented in Table 1. Statistical significance was set at $\mathrm{p}<0.05$.

\section{Results}

A total of 41 patients were admitted during this period with the diagnosis of aneurysmal SAH. 2 died within 30 days of admission. A further 2 inpatient mortalities were recorded and did not complete the 1-year follow-up period. 1 patient developed early seizures within 7 days despite prophylaxis and was excluded. The clinical characteristics of the patients included in the final analysis are displayed in Table 2.

Of the 36 patients who were included in the analysis, 21 suffered from an aneurysm in the anterior circulation and 15 in the posterior circulation. 21 patients had a modified Fisher grade of $\geq 3$ and 2 (6\%) patients were reported to have onset seizures with 1 patient subsequently developing a late seizure after discharge at 210 days. Most of the patients (94\%) were given Levetiracetam as 
Table 1. Overall seizure risk by clinical characteristics.

\begin{tabular}{|c|c|c|c|c|}
\hline & $\begin{array}{c}\text { No of patients } \\
\text { without seizures }\end{array}$ & $\begin{array}{l}\text { No of patients } \\
\text { with seizures }\end{array}$ & OR $(95 \% \mathrm{CI})$ & $\mathrm{p}$ Value \\
\hline GCS on arrival & 30 & 6 & $0.690(0.528,0.903)$ & 0.007 \\
\hline \multicolumn{5}{|c|}{ Total Duration of AED in hospital } \\
\hline$\leq 21$ days & 13 & 4 & Reference & \\
\hline$>21$ days & 17 & 2 & $0.382(0.060,2.421)$ & 0.307 \\
\hline $\begin{array}{l}\text { Proportion of Inpatient } \\
\text { Stay with AEDs }\end{array}$ & 30 & 6 & $0.964(0.934,0.994)$ & 0.019 \\
\hline \multicolumn{5}{|c|}{ Modified Fisher Grade } \\
\hline $0-2$ & 13 & 2 & Reference & \\
\hline $3-4$ & 17 & 4 & $1.529(0.242,9.675)$ & 0.652 \\
\hline \multicolumn{5}{|c|}{ Location of Aneurysm } \\
\hline Anterior circulation & 17 & 4 & Reference & \\
\hline Posterior circulation & 13 & 2 & $0.654(0.103,4.136)$ & 0.652 \\
\hline \multicolumn{5}{|c|}{ Vasospasms } \\
\hline No & 20 & 3 & Reference & \\
\hline Yes & 10 & 3 & $2(0.34,11.756)$ & 0.443 \\
\hline \multicolumn{5}{|c|}{ Hydrocephalus } \\
\hline No & 22 & 4 & Reference & \\
\hline Yes & 8 & 2 & $1.375(0.210,9.015)$ & 0.740 \\
\hline \multicolumn{5}{|c|}{ Intervention } \\
\hline Clip & 14 & 1 & Reference & \\
\hline Coil & 11 & 4 & $5.091(0.496,52.287)$ & 0.171 \\
\hline None & 5 & 1 & $2.800(0.146,53.709)$ & 0.495 \\
\hline
\end{tabular}

Table 2. Demographics and clinical characteristics.

\begin{tabular}{cc}
\hline Age, Mean +/- SD & Value (\%) \\
Male & $57+/-13$ \\
Female & $10(28)$ \\
Anterior Circulation & $26(72)$ \\
Posterior Circulation & \\
GCS $<13$ & $21(58)$ \\
Modified Fisher $\geq 3$ & $15(42)$ \\
$\quad$ & $21(58)$ \\
\hline
\end{tabular}




\section{Continued}

Complications

$\begin{array}{lc}\text { Onset seizures } & 2(6) \\ \text { Vasospasms } & 13(36) \\ \text { Hydrocephalus } & 10(28) \\ \text { Levetiracetam } & 34(94)\end{array}$

AED Prophylaxis

$\begin{array}{cc}\text { Phenytoin } & 2(6) \\ \text { Levetiracetam } & 34(94)\end{array}$

Median Duration of AED (IQR)

25 days $(13,48)$

Average Proportion of Inpatient Stay with AEDs

71

Intervention

Clipping
Coiling

No intervention

$6(16)$

Late Seizures

$>7$ days to 3 months

3

$>3$ months to 1 year

3

Mean time of onset of late seizures

128 days

Outcome

Median LOS

38 days

GCS $<15$ at discharge

$4(11 \%)$

prophylaxis and no delayed neurological deficits (DNID) were reported. 13 (36\%) patients were diagnosed with vasospasms and 10 (28\%) developed hydrocephalus during their stay. Median LOS was 38 days with median duration of AED administered at 25 days. Majority of the patients made a full neurological recovery with only 4 patients (11\%) having a GCS of $<15$ at discharge.

3 patients developed delayed seizures between 14 days to 3 months and 3 other patients presented with late seizures beyond 3 months to a year of follow-up. In total, 6 (17\%) patients developed late onset seizures. Mean time of onset of late seizures was 128 days (4.3 months).

Patients with a better GCS on arrival had a lower risk of developing late seizures $(\mathrm{OR}=0.690,95 \% \mathrm{CI}=(0.528,0.903), \mathrm{p}=0.007)$. Those patients who received a greater proportion of inpatient stay with AED prophylaxis had a statistically significant lower risk of developing late seizures $(\mathrm{OR}=0.964,95 \% \mathrm{CI}=$ (0.934, 0.994), $\mathrm{p}=0.019)$. Although the value tended towards benefit $(\mathrm{OR}=$ 
$0.38295 \% \mathrm{CI}=(0.060,2.421))$ for AED $>21$ days in hospital, the result was not statistically significant $(\mathrm{p}=0.307)$.

\section{Discussion}

The purpose of this study is to examine our local experience with extended duration of AED prophylaxis in relation to late onset of seizures. We also analyzed the various interventions and known risk factors that are associated with late seizures that often progress to chronic epilepsy.

Seizures in aneurysmal SAH are predominantly simple partial seizures with or without secondary generalization. Non-convulsive status epilepticus has been reported in up to $18 \%$ of cases [2] [14] [15]. Diagnosis will require a high index of suspicion and performance of an EEG. Routine seizure prophylaxis with AEDs remains controversial as there is a lack of demonstrable benefit [16] [17]. Phenytoin was the predominant AED in these studies and there was considerable concern over their routine use due to adverse impact on outcome. Phenytoin was identified as an independent predictor for poor functional outcome at 14 days (OR 1.5\%, 95\% CI 1.2 - 1.9) and for poor cognitive outcome at 3 months ( $p$ $=0.003$ ) [9]. The use of AEDs in patients with aneurysmal SAH was also associated with poor 3-month outcome with worse Glasgow outcome scale, vasospasms, neurological deterioration, cerebral infarction and fever [8].

On the other hand, some studies have demonstrated increased risk of late seizures in patients who are not started on AED prophylaxis compared to those who were treated early [7] [18]. More recent studies with Levetiracetam as the main drug suggest lower seizure incidence and better functional outcomes at discharge as compared to traditional AEDS [19] [20].

A separate study by Murphy-Human $\mathrm{T}$ et al. comparing extended course of phenytoin with a 3-day course of Levetiracetam in 442 patients found a higher rate of in-hospital seizures in the latter group (3.4\% vs $8.3 \%$ ) [21]. The authors attributed most of this difference to late seizures in the Levetiracetam group and suggested a longer duration of prophylaxis may be required to minimize seizures. Considering this finding, the same authors conducted the first randomized trial evaluating duration of seizure prophylaxis after SAH by comparing the effectiveness of a short 3-day course of Levetiracetam to an extended course of the same drug till discharge [22]. In-hospital seizures occurred in 3 (9\%) in the short course versus $1(2 \%)$ in the extended group. Although superiority of extended duration of Levetiracetam was not demonstrated, there was a trend to benefit in reducing in-hospital seizures.

After retrospectively reviewing our own group of patients, we found a statistically significant difference in the OR for late seizures in patients who received a higher proportion of inpatient stay with AED prophylaxis as compared to those who received a lower proportion $(\mathrm{p}=0.019)$. However, the absolute OR is small (0.964) and its impact on clinical significance remains to be further investigated. A 21-day cut-off point, which was the upper limit described in the study by De- 
wan MC et al., [12] was applied in our data analysis. The OR for patients on extended duration of AEDs $>21$ days trended towards benefit (0.382), but the result was not statistically significant $(\mathrm{p}=0.307$ ). It would, nonetheless, be difficult to recommend routine till-discharge prescription of AED due to increased cost and they should only be reserved for patients who fulfil a predefined set of clinical criteria. Other factors examined such as modified Fisher scale, presence of vasospasms or hydrocephalus did not yield statistically significant results. Most of our patients received Levetiracetam and it was generally well tolerated with no reported clinically significant side effects or DNID.

\section{Limitations}

Our study has some limitations due to its retrospective design. The diagnosis of seizures was mostly clinical except for one via EEG. Routine EEG monitoring was not performed in all subjects and this may lead to under-reporting of seizures, especially non-convulsive seizures. Although our electronic medical records system is robust and covers nationwide data, side effect of AEDs may have been under-recorded. Other limitations include a smaller final sample size due to mortalities during the long follow-up period specified in our study. Given the difficulty in recruitment for randomized trials [19], a large multi-centered study will be required to determine the optimum duration of Levetiracetam as well as specific criteria for extended duration of AED prophylaxis.

\section{Conclusion}

Patients who had a larger proportion of inpatient stay on AED prophylaxis had a statistically significant reduction in risk of developing late seizures. Patients who received more than 21 days of AEDS had an OR (0.382) that trended towards benefit but was not statistically significant. The optimum duration of AED prophylaxis, specifically Levetiracetam and the clinical criteria for recommendation of extended duration of AEDs to prevent late onset seizures will require further large multi-centered randomized studies.

\section{Previous Presentation}

Not applicable.

\section{Conflicts of Interest}

No potential conflict of interest relevant to this article was reported.

\section{Funding}

No funding was received.

\section{Data Availability}

Original encrypted database is available in organization's computer storage system. 


\section{References}

[1] Choi, K.S., Chun, H.J., Yi, H.J., Ko, Y., Kim, Y.S. and Kim, J.M. (2009) Seizures and Epilepsy following Aneurysmal Subarachnoid Hemorrhage: Incidence and Risk Factors. Journal of Korean Neurosurgical Society, 46, 93-98.

https://doi.org/10.3340/jkns.2009.46.2.93

[2] Lin, C.L., Dumont, A.S., Lieu, A.S., Yen, C.P., Hwang, S.L., Kwan, A.L., et al. (2003) Characterization of Perioperative Seizures and Epilepsy Following Aneurysmal Subarachnoid Hemorrhage. Journal of Neurosurgery, 99, 978-985. https://doi.org/10.3171/jns.2003.99.6.0978

[3] Lin, Y.J., Chang, W.N., Chang, H.W., Ho, J.T., Lee, T.C., Wang, H.C., et al. (2008) Risk Factors and Outcome of Seizures after Spontaneous Aneurysmal Subarachnoid Hemorrhage. European Journal of Neurology, 15, 451-457. https://doi.org/10.1111/j.1468-1331.2008.02096.x

[4] Kvan, D.A., Loftus, C.M., Copeland, B. and Quest, D.O. (1983) Seizures during the Immediate Post-Operative Period. Neurosurgery, 12, 14-17. https://doi.org/10.1227/00006123-198301000-00003

[5] Butzkueven, H., Evans, A.H., Pitman, A., Leopold, C., Jolley, D.J., Kaye, A.H., et al. (2000) Onset Seizures Independently Predict Poor Outcome after Subarachnoid Hemorrhage. Neurology, 55, 1315-1320. https://doi.org/10.1212/WNL.55.9.1315

[6] Kanner, A.M. (2003) Subarachnoid Hemorrhage as a Cause of Epilepsy. Epilepsy Currents, 3, 101-102. https://doi.org/10.1046/j.1535-7597.2003.03310.x

[7] Raper, D.M., Starke, R.M., Komotar, R.J., Allan, R., Connolly Jr., E.S. (2013) Seizures after Aneurysmal Subarachnoid Hemorrhage: A Systematic Review of Outcomes. World Neurosurgery, 79, 682-690.

https://doi.org/10.1016/j.wneu.2012.08.006

[8] Rosengart, A.J., Huo, J.D., Tolentino, J., Novakovic, R.L., Frank, J.I., Goldenberg, F.D., et al. (2007) Outcome in Patients with Subarachnoid Hemorrhage Treated with Antiepileptic Drugs. Journal of Neurosurgery, 107, 253-260.

https://doi.org/10.3171/JNS-07/08/0253

[9] Naidech, A.M., Kreiter, K.T., Janjua, N., Ostapkovich, N., Parra, A., Commichau, C., et al. (2005) Phenytoin Exposure Is Associated with Functional and Cognitive Disability after Subarachnoid Hemorrhage. Stroke, 3, 583-587. https://doi.org/10.1161/01.STR.0000141936.36596.1e

[10] Shah, D. and Husain, A.M. (2009) Utility of Levetiracetam in Patients with Subarachnoid Hemorrhage. Seizure, 18, 676-679.

https://doi.org/10.1016/j.seizure.2009.09.003

[11] Nau, K.M., Divertie, G.D., Valentino, A.K. and Freeman, W.D. (2009) Safety and Efficacy of Levetiracetam for Critically Ill Patients with Seizures. Neurocritical Care, 11, 34-37. https://doi.org/10.1007/s12028-009-9185-0

[12] Dewan, M.C. and Mocco, J. (2015) Current Practice Regarding Seizure Prophylaxis in Aneurysmal Subarachnoid Hemorrhage across Academic Centers. Journal of NeuroInterventional Surgery, 7, 146-149. https://doi.org/10.1136/neurintsurg-2013-011075

[13] World Health Organization (2018) International Classification of Diseases for Mortality and Morbidity Statistics. 11th Revision.

[14] Marigold, R., Günther, A., Tiwari, D. and Kwan, J. (2013) Antiepileptic Drugs for Primary and Secondary Prevention of Seizures after Subarachnoid Hemorrhage. Cochrane Systematic Review, No. 6, CD008710. 
https://doi.org/10.1002/14651858.CD008710.pub2

[15] Claassen, J., Perotte, A., Albers, D., Kleinberg, S., Schmidt, J.M., Tu, B., et al. (2013) Nonconvulsive Seizures after Subarachnoid Hemorrhage: Multimodal Detection and Outcomes. Annals of Neurology, 74, 53-64. https://doi.org/10.1002/ana.23859

[16] Rhoney, D.H., Tipps, L.B., Murry, K.R., Basham, M.C., Michael, D.B. and Coplin, W.M. (2000) Anticonvulsant Prophylaxis and Timing of Seizures after Aneurysmal Subarachnoid Hemorrhage. Neurology, 55, 258-265. https://doi.org/10.1212/WNL.55.2.258

[17] Panczykwoski, D., Pease, M., Zhao, Y., Weiner, G., Ares, W., Crago, E., et al. (2016) Prophylactic Antiepileptics and Seizure Incidence Following Subarachnoid Hemorrhage: A Propensity Score-Matched Analysis. Stroke, 47, 1754-1760. https://doi.org/10.1161/STROKEAHA.116.013766

[18] Heros, R.C. (2007) Antiepileptic Drugs and Subarachnoid Hemorrhage. Journal of Neurosurgery, 107, 251-252. https://doi.org/10.3171/JNS-07/08/0251

[19] Szaflarski, J.P., Meckler, J.M., Szaflarski, M., Shutter, L.A., Privitera, M.D. and Yates, S.L. (2007) Levetiracetam Use in Critically Ill Patients. Neurocritical Care, 7, 140-147. https://doi.org/10.1007/s12028-007-0042-8

[20] Mink, S., Muroi, C., Seule, M., Bjeljac, M. and Keller, E. (2011) Levetiracetam Compared to Valproic Acid: Plasma Concentration Levels, Adverse Effects and Interactions in Aneurysmal Subarachnoid Hemorrhage. Clinical Neurology and Neurosurgery, 113, 644-648. https://doi.org/10.1016/j.clineuro.2011.05.007

[21] Murphy-Human, T., Welch, E., Zipfel, G., Diringer, M.N. and Dhar, R. (2011) Comparison of Short-Duration Levetiracetam with Extended-Course Phenytoin for Seizure Prophylaxis after Subarachnoid Hemorrhage. World Neurosurgery, 75, 269-274. https://doi.org/10.1016/j.wneu.2010.09.002

[22] Human, T., Diringer, M.N., Allen, M., Zipfel, G.J., Chicoine, M., Dacey, R., et al. (2018) A Randomized Trial of Brief versus Extended Seizure Prophylaxis after Aneurysmal Subarachnoid Hemorrhage. Neurocritical Care, 28, 169-174. https://doi.org/10.1007/s12028-017-0440-5 